\title{
Le cabinet médical en SA ou en Sàrl
}

Dominique Sierro, secrétaire général de la SMV
Correspondance: Dominique Sierro Avocat et notaire Place de la Gare 2 CH-1951 Sion

Tél. 0273232424

sierro@avocatnotaire.ch
De plus en plus de médecins se demandent quelle forme juridique ils doivent donner à leur cabinet médical. Si, jusqu'à aujourd'hui, la quasitotalité des médecins avaient adopté la forme de la raison individuelle ou de la société simple, la question de savoir s'il est permis, voire opportun de pratiquer sous la forme de la société anonyme (SA), cas échéant, de la société à responsabilité limitée (Sàrl), se pose avec acuité.

Rappelons tout d'abord que la forme juridique de la raison individuelle existe dès le moment où le médecin exerce seul son activité, à titre indépendant.

Lorsque plusieurs médecins conviennent de s'associer afin de partager les frais d'exploitation du cabinet, d'engager du personnel commun, de louer ensemble des locaux, etc., ils forment une société simple au sens des articles 530ss du Code des Obligations (CO). Cette société se confond avec un contrat, car elle n'a pas d'existence juridique propre, à savoir qu'elle n'a ni la jouissance, ni l'exercice des droits civils, ni la possibilité d'agir en justice. Dans ce cas, il est recommandé de signer une convention en bonne et due forme afin de préciser les apports de chacun, les droits et obligations de chaque associé, etc.

Théoriquement, il est également possible d'envisager la constitution d'une société en commandite, d'une coopérative ou même d'une société en nom collectif. Ces formes juridiques ne sont néanmoins pas très courantes et peu usitées dans le domaine médical.

\section{Qu'en est-il alors de la forme juridique de la SA ou de la Sàrl?}

La LAMal a levé l'interdiction faite à un médecin, admis à pratiquer à la charge de l'assurance obligatoire, de se trouver dans un rapport de dépendance d'une société, en dehors des hôpitaux.

La FMH, quant à elle, tolère ce genre d'organisation, notamment depuis qu'elle a signé la convention-cadre TARMED, en date du 5 juin 2002. En effet, l'article 9 de dite convention traite de l'engagement de médecins et de collaboration sous la forme juridique d'une personne morale. L'alinéa 3 prévoit ce qui suit: la collaboration sous la forme juridique d'une personne morale (SA, Société en commandite, Sàrl, Coopérative, Association, etc.) est possible. Dans cette disposition, il est également précisé que les pres- tations fournies doivent pouvoir être attribuées aux divers médecins au moyen du numéro EAN de manière telle qu'il ressorte de la facture quel est le médecin principalement responsable des prestations fournies. La convention réserve aussi la légalisation cantonale.

Relevons d'emblée que le fait qu'un médecin donne à son cabinet la forme de la SA ou de la Sàrl ne modifie en rien ses obligations professionnelles. Il demeure personnellement responsable des prestations prodiguées. Quant au patient lésé, il peut, en tout temps, faire valoir ses droits contre un médecin fautif, même si celui-ci est employé d'une SA ou d'une Sàrl. Le Code de déontologie de la FMH rappelle aussi clairement, à son article 9, que le médecin doit veiller, dans la mesure du possible, à assurer la continuité de l'assistance personnelle, que son activité soit indépendante ou salariée. Le même article prévoit que, dans le cadre de son activité salariée, le médecin ne peut exercer en son propre nom mais qu'il doit veiller à ce que le patient sache clairement à qui il confie son mandat thérapeutique.

\section{Comment, dès lors, constituer une SA ou une Sàrl?}

La SA est une société dite de capitaux. Les fondateurs et actionnaires ne sont pas liés, en principe, par des liens juridiques personnels.

Seules leurs participations financières sont associées au but de la société. Autrement dit, selon l'article $620 \mathrm{CO}$, la SA est celle qui se forme sous une raison sociale dont le capital-actions est déterminé à l'avance, divisé en actions et dont les dettes ne sont garanties que par l'actif social. Dès son inscription au Registre du commerce, la société acquiert la personnalité juridique. Dès ce moment, elle a la jouissance et l'exercice des droits civils et est reconnue comme une personne morale.

Lors de sa constitution, la société doit avoir au moins 3 membres fondateurs. Le capital-actions minimum de la société est de Fr. 100 000.-. S'il n'est pas entièrement libéré, $20 \%$, mais au moins Fr. 50 000.-, doivent l'être le jour de la constitution. Les actions peuvent être nominatives ou au porteur. Sur le plan de l'organisation de la société, l'entité compte obligatoirement 3 organes qui sont l'assemblée générale, pouvoir suprême, 
le conseil d'administration, organe exécutif à qui sont dévolues toutes les tâches qui ne sont pas expressément de la compétence de l'assemblée générale et l'organe de révision, chargé du contrôle de la gestion comptable de la société.

Quant à la Sàrl, elle est la société que forme 2 ou plusieurs personnes sous une raison sociale et dont le capital-social est d'au moins Fr. 20 000.-, divisé en parts sociales de Fr. 1000.- ou d'un multiple de 1000. Le capital-social ne peut excéder Fr. 2000 000.-. Dans tous les cas, 50\% du capital doivent être libéré. A la différence de la SA, tout associé répond des engagements de la société au-delà de sa part sociale, mais au plus à concurrence du capital-social inscrit, à moins que la libération entière du capital ne puisse être prouvée et que celui-ci n'a pas été indûment remboursé aux associés. En d'autres termes, si le capital-social a été effectivement libéré, les associés n'encourent pas de responsabilité personnelle.

Contrairement à la SA, la Sàrl est empreinte de plusieurs aspects des sociétés de personnes. La venue d'un nouvel associé nécessite la passation d'un acte authentique devant notaire. Les statuts peuvent prévoir une clause de prohibition de concurrence. Chaque associé participe, en principe, à la gestion de la société. Sur le plan de l'organisation de la Sàrl, l'entité compte obligatoirement 2 organes qui sont l'assemblée des associés, pouvoir suprême et les gérants, organe exécutif de la société. Contrairement aux droits de la SA, l'organe de révision est facultatif.

Enfin, en raison du fait que la loi n'exige pas, pour la direction de la Sàrl, une majorité d'asso- ciés suisses, domiciliés en Suisse (comme c'est le cas pour le conseil d'administration d'une SA), cette forme de société convient, en particulier, à des personnes de nationalité étrangère désirant exploiter une activité en Suisse. Relevons, toutefois, que le droit de la Sàrl est actuellement en révision et que certains points mentionnés cidessus vont être modifiés.

\section{Quels sont, en fait, les avantages et inconvénients de la SA ou de la Sàrl?}

Divers critères permettent d'évaluer le bienfondé du choix d'une forme juridique. Il y a tout d'abord le choix personnel du médecin fondé, notamment, sur son expérience. Dans la pratique, on examinera surtout les critères de coûts de fonctionnement (charges fiscales et sociales, charges de gestion comptable), de responsabilité, d'optimisation fiscale, de transfert de cabinet (cession contractuelle, succession) et de frais de constitution. Ces aspects sont traités dans l'article suivant de Jean-Pierre Chevalley.

En conclusion, il est bien loin le temps où certains médecins ne voyaient dans l'établissement de leur cabinet sous la forme de la SA ou de la Sàrl qu'une démarche matérialiste. En réalité, la tendance au regroupement est inéluctable pour optimiser l'offre de prestations, mais également afin de gagner en qualité de vie (répartition du travail, permanence, etc.) ou de répondre à des objectifs de pérennité du cabinet. Tout médecin, qu'il soit en fin ou en début de carrière, devrait y songer sérieusement! 


\section{Avantages et inconvénients d'exercer son activité de médecin sous la forme d'une SA ou d'une Sàrl?}

\section{Jean-Pierre Chevalley}

Correspondance: Jean-Pierre Chevalley FMH Fiduciaire Services Avenue des Alpes 6 Case postale 391 $\mathrm{CH}-3960$ Sierre Tél. 0274551675 Fax 0277232001 fmhfiduciaire.ch
Les avantages pour le médecin d'exercer sous la forme d'une société anonyme (SA) ou d'une société à responsabilité limitée (Sàrl) sont multiples:

- responsabilité limitée au capital;

- gain en capital exonéré si vente d'actions;

- planification dans le cadre de la succession;

- structure claire entre la fortune commerciale et la fortune privée;

- optimisation de la charge fiscale, etc.

Avant de passer en SA ou Sàrl, il faut bien peser les avantages et les inconvénients d'une telle décision. En effet, un cabinet en SA ou Sàrl a des obligations légales, comptables et fiscales plus importantes que les obligations qui incombent au médecin indépendant. Tout d'abord, le médecin doit supporter les frais de fondations de la nouvelle société. Précisons tout de même que, pour une simple Sàrl d'un capital-action de Fr. 20 000.-, on se situe en dessous de Fr. 2000.-. Ensuite, de multiples démarches administratives doivent être entreprises lors du changement de la forme juridique, les contrats de location ou de travail doivent être transférés au nom de la société, par exemple. D'un autre côté, nous pouvons tenir compte de l'abattement de $50 \%$ sur le bénéfice de la vente du cabinet pour l'impôt cantonal et communal (art. 63, al. 3 LF). Les frais de fonctionnement sont également plus élevés; en effet, une SA doit faire appel à un organe de révision, en général une fiduciaire, ce qui engendre des coûts supplémentaires. Ceci n'est pas nécessaire pour une Sàrl. Toutefois, la loi sur la révision a été modifiée (art. $698 \mathrm{CO}$ ) et à partir de 2008, les petites entreprises pourront renoncer à ce contrôle. Un désavantage de la SA est la double imposition du bénéfice. Si le bénéfice de l'exercice est de Fr. 100 000.- et qu'ensuite le dividende est distribué aux actionnaires, l'impôt sera payé à la fois par la société et par les actionnaires. Or, dans un cabinet en SA ou Sàrl, le médecin perçoit un salaire et, en général, il n’y a pas de distribution de bénéfice.

Quel est l'impact des avantages énumérés plus haut pour le médecin désirant transformer son cabinet en personne morale?

\section{Responsabilité limitée au capital}

La responsabilité financière est limitée à la libération du capital, mais le médecin reste toujours responsable envers ses patients de l'exécution du contrat de mandat qui lui est confié (cf. l'article de Me Dominique Sierro, Secrétaire général de la SMV).

\section{Gain en capital exonéré en cas de vente d'actions}

En cas de revente des actions de la SA, la valeur des actions est déterminante. Le goodwill, étant compris dans la valeur des actions, ne sera donc pas imposé.

\section{Planification dans le cadre de la succession}

Pour des raisons d'ordre familial par exemple, le médecin désire laisser une partie de son revenu dans la société anonyme, étant supposé qu'en cas de divorce, il pourrait envisager une diminution de la pension alimentaire.

La fortune de la société ne sera pas touchée ce qui permettra au médecin de continuer son activité en toute sérénité n'ayant à remettre dans la succession qu'une partie de ses actions (il devrait rester majoritaire).

Dans le cadre de la «réforme de l'imposition des entreprises II», il est prévu que les dividendes provenant d'une participation faisant partie de la fortune privée soient imposés à hauteur de $80 \%$, voire moins. Cette réforme devrait, selon toute vraisemblance, entrer en vigueur au $1^{\text {er }}$ janvier 2007, voire

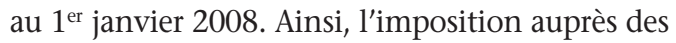
actionnaires serait atténuée de $20 \%$ et plus.

\section{Structure claire entre la fortune commerciale et la fortune privée:}

Pour éviter que le cabinet médical prenne une place prépondérante dans la fortune commerciale, on peut imaginer que ce dernier demeure la propriété personnelle du médecin. L'imposition, en cas de revente, serait régie par les dispositions sur les gains immobiliers et non par celles relatives au revenu ordinaire; elle ne sera pas non plus soumise aux cotisations AVS 


\section{Optimisation de la charge fiscale}

Le revenu du médecin indépendant, qui appartient en général à la classe des hauts revenus, est aujourd'hui ponctionné à la hauteur d'un taux marginal de $40 \%$ du revenu imposable. A cela il faut ajouter 9,5\% du revenu perçu par l'AVS, soit une charge totale de 50\%. Avec le décret modifiant la loi fiscale du 17 juin 2005, le canton du Valais est devenu, depuis le $1^{\text {er }}$ janvier 2006, le $4^{\mathrm{e}}$ canton fiscalement le plus attractif de Suisse. La principale mesure d'allègement fiscal apportée pour renforcer les conditions cadres favorables au PME est la réduction du taux d'impôt sur le bénéfice des personnes morales. En effet, celui-ci baisse de 4 à $3 \%$ jusqu'à un montant de Fr. 100 000.- au lieu de Fr. 30 000.- précédemment. Il y a lieu de mentionner une diminution sur le capital de 1,5 à 1\%o jusqu'à Fr. 500 000.de capital imposable. Il faut également relever l'introduction d'une mesure tout à fait exceptionnelle sur le plan fiscal. Il s'agit, en effet, d'admettre la possibilité d'un amortissement immédiat et jusqu'à 50\% de tout nouvel investissement, à l'exception des immeubles. En ce qui concerne les immeubles lors de la construction ou de l'achat, le taux ordinaire d'amortissement peut être doublé.

En admettant que le médecin désire laisser une partie des bénéfices de l'exercice dans son entreprise, ce montant ne sera imposé qu'à envi- ron $15 \%$ pour les premiers Fr. 100 000.-, il pourra également, dans le cas où il est propriétaire des locaux utilisés par le cabinet, percevoir un loyer, lequel ne sera pas soumis à l'AVS.

Le médecin qui aura transformé son cabinet en société anonyme sera considéré comme salarié de celle-ci. Il n'aura plus qu'à couvrir la moitié de ses charges salariales, le reste étant pris en charge par la société. Cela permettra également de mieux coordonner sa couverture en assurances invalidité, maladie et accident.

Le statut de salarié lui permettra également d'adhérer au $2^{\mathrm{e}}$ pilier, où des rachats seront possibles, afin d'alléger sa fiscalité. Le médecin indépendant peut aussi s'affilier à ce pilier, mais il doit prendre à sa charge l'intégralité des cotisations.

\section{«Penser globalement, agir localement»}

Ce slogan est particulièrement pertinent dans le domaine de la fiscalité. En effet, il est nécessaire d'aborder ce problème globalement pour mesurer les interactions de divers facteurs: engagements financiers, fortune, assurance, régime matrimonial, etc. Cette planification coordonnée permet d'acquitter l'impôt dans les conditions les plus favorables. C'est pourquoi un check-up approfondi de la situation du médecin doit être effectué, afin d'utiliser au mieux les divers instruments financiers. 